bioRxiv preprint doi: https://doi.org/10.1101/847608; this version posted November 20, 2019. The copyright holder for this preprint (which was not certified by peer review) is the author/funder, who has granted bioRxiv a license to display the preprint in perpetuity. It is made available under aCC-BY-ND 4.0 International license.

\title{
COLD AND DISTANT: STRUCTURAL FEATURES OF THE NUCLEOPROTEIN COMPLEX OF A COLD-ADAPTED INFLUENZA A VIRUS STRAIN
}

Shvetsov A.V.a,b,c, Lebedev D.V.a,c, Zabrodskaya Y.A. ${ }^{\text {ab,c,d, }}$, Shaldzhyan A.A.a,d, Egorova M.A.d, Vinogradova D.S.,e, Konevega A.L. a,b,c, Gorshkov A.N. d, Ramsay E.S. ${ }^{\mathrm{d}}$, Radulescu A.f , Sergeeva M.V. d, Plotnikova M.A.d, Komissarov A.B. ${ }^{\mathrm{d}}$, Taraskin A.S. ${ }^{\mathrm{d}}$, Lebedev K.I. ${ }^{\mathrm{d}, \mathrm{g}}$, Garmay Yu.P. ${ }^{\mathrm{a}}$, Kuznetsov V.V. ${ }^{\mathrm{d}}$, Isaev-Ivanov V.V. ${ }^{\mathrm{a}}$, Vasin A.V.b,d,h, Tsybalova L.M. ${ }^{\mathrm{d}}$, Egorov V.V.a,c,d,i

\begin{abstract}
a Petersburg Nuclear Physics Institute named by B. P. Konstantinov of National Research Center "Kurchatov Institute", 188300, mkr. Orlova roshcha 1, Gatchina, Russia

b Peter the Great Saint Petersburg Polytechnic University, 194064, Politekhnicheskaya 29, St. Petersburg, Russia

c National Research Centre Kurchatov Institute, 123182, Akademika Kurchatova Sq. 1, Moscow, Russia

d Smorodintsev Research Institute of Influenza, Russian Ministry of Health, 197376, Prof. Popov 15/17, St. Petersburg, Russia

e NanoTemper Technologies Rus, 191167, Alexandra Nevskogo 9, office 312, St. Petersburg, Russia

f Juilich Centre for Neutron Science at Heinz Maier-Leibnitz Zentrum, Lichtenbergstr. 1, D-85747 Garching, Munich,

Germany

g Pavlov First Saint Petersburg State Medical University, 197022, L'va Tolstogo 6-8, St. Petersburg, Russia

${ }^{\text {h }}$ St. Petersburg State Chemical-Pharmaceutical Academy, 197022, Prof. Popov 14A, St. Petersburg, Russia

i Federal State Budgetary Scientific Institution "Institute of Experimental Medicine", 197376, Akademika Pavlova 12, St.

Petersburg, Russia
\end{abstract}

\section{Corresponding author:}

Dr. Yana A Zabrodskaya

yana@zabrodskaya.net; zabrodskaya_ya@pnpi.nrcki.ru $188300, \mathrm{mkr}$. Orlova roshcha 1 , Gatchina, Russia

Petersburg Nuclear Physics

Institute named by B. P.

Konstantinov of National Research

Center "Kurchatov Institute",

Molecular and Radiation Biophysics

Department

\section{Keywords:}

nucleoprotein;

molecular dynamics;

cold-adapted strain;

influenza A;

E292G

\begin{abstract}
Two influenza A nucleoprotein variants (wt: G102R; and mutant: G102R and E292G) were studied with regard to macro-molecular interactions in oligomeric form (24-mers). The E292G mutation has been previously shown to provide cold adaptation. Molecular dynamics simulations of these complexes and trajectory analysis showed that the most significant difference between the obtained models was distance differences between nucleoprotein complex strands. Influenza virus nucleoprotein complexes were isolated from strains bearing the corresponding NP amino acid substitutions. The isolated complexes were characterized by transmission electron microscopy and differential scanning fluorimetry (DSF). Presence of the E292G substitution was shown by DSF to affect nucleoprotein complex melting temperature. In the filament interface peptide model, it was shown that the peptide corresponding in primary structure to the wild-type NP (SGYDFEREGYS, wild type peptide) is prone to temperature-dependent selfassociation, unlike the peptide carrying the substitution corresponding to E292G (SGYDFGREGYS, mutant peptide). It was also shown that the SGYDFEREGYS peptide (wt) is capable of interacting with a recombinant full-size monomeric nucleoprotein (with primary structure corresponding to wild type); this interaction's equilibrium dissociation constant is five orders of magnitude lower than for the SGYDFGREGYS peptide. Using small-angle neutron scattering (SANS), the supramolecular structures of isolated complexes of these proteins was studied at temperatures of 15,32 , and $37^{\circ} \mathrm{C}$. SANS data show that the structures of the studied complexes (mutant or normal proteins with RNA) at elevated temperature differ from the rod-like particle model and react differently to temperature changes. The data suggest that the mechanism behind cold adaptation with E292G is associated with a weakening of the interaction between strands of the ribonucleoprotein complex and, as a result, the appearance of interchain interface flexibility necessary for complex function at low temperature.
\end{abstract}

\section{Introduction}

The incidence of influenza is detrimental to human health and is a serious economic concern. Vaccination is one of the most effective ways to prevent the occurrence of this illness [1]. It is advisable to vaccinate using live attenuated vaccines [2], including vaccines created using cold-adapted, temperature-sensitive strains as donors of attenuation [3]. The NP protein is one of eight major structural proteins of the influenza virus [4]. As a ribonucleoprotein particle component, it takes part in both the packaging of viral genetic 
material and in replication [5]. In the vaccine strain obtained during cold adaptation of the influenza A/HK/68 virus, a mutation leading to the amino acid substitution E292G in the NP protein was detected [3]. This substitution is sufficient for the virus to acquire a cold adaptation phenotype. The presence of another substitution (G102R), however, is not related to the acquisition of this property directly, but is necessary for the virus to multiply when the E292G substitution is present [6]. The molecular mechanisms behind the acquisition of cold adaptation, through these substitutions, remain unclear. Elucidation of such mechanisms is necessary for rational design of new strains, including donors of attenuation necessary for the creation of new live attenuated vaccines. The presence of certain substitutions in NP (G102R, E292G) leads to cold adaptation, but not temperature sensitivity. E292G was shown [6] in vitro to be insufficient to confer temperature sensitivity. Moreover, E292G is also seen in vivo. It has been detected in viruses infecting wild birds (whose body temperatures are commonly $39^{\circ} \mathrm{C}$ and above).

In this research, we study the influence of the substitutions G102R and E292G on ribonucleoprotein complex structure using molecular dynamics, differential scanning fluorimetry, and small-angle neutron scattering.

\section{Materials and methods \\ Molecular Modeling}

To build NP filaments models, we used existing cryoelectron microscopy and initial model data available from pdbid:4bbl [5]. Full monomer models were built using the Modeler software package and pdbid:2iqh [7] as template. Initial structure was modified as follows: wild-type NP contains the substitution G102R; and mutant (cold-adapted) NP contains two substitutions simultaneously (G102R and E292G). Next, each monomer was fitted to the $4 \mathrm{bbl}$ structure. Then, RNA was added using $\mathrm{P}$ atoms trace in 4bbl. RNA was built using the Ambertools package. The resulting structure was then used for iterative optimization using Pymol [8] and GROMACS [9] to resolve all clashes.

\section{Molecular dynamics}

All MD simulations were performed in the GROMACS [9], [10] software package using an amber99sb-ildn-bsc0 [11], [12] force field for protein and RNA, and a tip3p [13] model for explicit water. The simulated systems were around $2.6 \mathrm{M}$ atoms in size, including an explicit solvent shell and $50 \mathrm{mM} \mathrm{NaCl}$ to neutralize charges. Solvent shell thickness was at least $2.0 \mathrm{~nm}$. Box size was around 50x22x24 nm. Systems were simulated at two temperatures, $299 \mathrm{~K}$ and $312 \mathrm{~K}$. For each temperature, systems were equilibrated using a two-step protocol. During the first step, the system was equilibrated for $5 \mathrm{~ns}$ with all heavy, non-solvent atoms restrained to their initial positions using NPT ensemble. During the second step, systems were equilibrated without restraints for $10 \mathrm{~ns}$ (starting from the last frame of the previous step). After two equilibration stages, a 250 ns trajectory was simulated with a time step of 2 fs. A Neighbor search was performed every 50 steps. The PME algorithm was used for modeling electrostatic and Van der Waals interactions with a cutoff of $1.2 \mathrm{~nm}$. Temperature coupling was done with the Nose-Hoover algorithm. Pressure coupling was done with the ParrinelloRahman algorithm for 1 bar. All further analyses were performed on the final 100 ns time interval.

\section{RNPs}

Isolation of influenza A H3N2 virus (A/Hong Kong/68) ribonucleoprotein complexes was carried out using a viral suspension kindly provided by the Smorodintsev Research Institute of Influenza's Influenza Vaccine Laboratory, following published methods [14]. Identification of the NP-containing fractions was carried out using described Western blotting methods [15]. For SANS, the protein was transferred to a heavy water buffer. Two hundred microliters of mix (combined, NP-containing pooled glycerol fraction mix, purity above $95 \%$ ) was applied to a micro-centrifuge filter (with a pore size corresponding to a molecular weight of $100 \mathrm{kDa}$ ) and centrifuged until the volume was reduced to $50 \mu \mathrm{l}$. Next, $150 \mu \mathrm{l}$ of PBS (in $\mathrm{D}_{2} 0$ ) was added and the centrifugation wash procedure repeated 3 times.

\section{TEM}

For transmission electron microscopy, a suspension of the isolated nucleoprotein complexes at a concentration of $1 \mathrm{mg} / \mathrm{ml}$ was deposited on copper electron microscopy grids coated with a carbon substrate. After adsorption of RNP complexes to the substrate for $1 \mathrm{~min}$, the grids were washed twice with distilled water. Further, negative contrasting of the samples on grids with a $2 \%$ solution of sodium salt of phosphoric tungsten acid was performed for $1 \mathrm{~min}$. After contrasting, grids were dried and examined using a JEOL JEM 1011 transmission electron microscope at an accelerating voltage of $80 \mathrm{kV}$. Electron micrographs were taken using a Morada digital camera (Olympus, 
Inc). The procedure was repeated 5 times.

\section{DSF}

Thermal protein denaturation stability analysis was performed via differential scanning fluorimetry (using intrinsic fluorescence of tryptophan residues) by slowly heating the sample in a Prometheus NT.48 scanning fluorimeter (NanoTemper Technologies, $\mathrm{GmbH}$ ). Nucleoprotein samples in PBS buffer solution (at concentrations of $3 \mathrm{mg} / \mathrm{ml}$ ) were loaded into quartz capillaries (Standard Capillaries Prometheus NT.48, NanoTemper); capillaries were placed on the platform and measurements were taken in the temperature range from $15^{\circ} \mathrm{C}$ to $95^{\circ} \mathrm{C}$ in increments of $1{ }^{\circ} \mathrm{C} / \mathrm{min}$ at a laser intensity of $50 \%$. Fluorescence intensities were recorded at $330 \mathrm{~nm}$ and $350 \mathrm{~nm}$; and the ratio of the fluorescence intensities and the first derivative were analyzed.

\section{SPR}

Measurements were taken using a GE Healthcare Biacore X100 instrument in accordance with the manufacturer's instructions. Commercial NP protein
(H3N2) was covalently immobilized on CM5 chips (GE Healthcare, USA) and used as a ligand. As a result of optimization of conditions, NP immobilization was carried out in a $10 \mathrm{mM}$ MES buffer, $\mathrm{pH}$ 6.0. Solutions of the SGYDFEREGYS and SGYDFGREGYS peptides in PBST buffer (GE Healthcare, USA) at the indicated concentrations were used as analyte. Data were processed in the Biacore X100 software and visualized in Origin2015.

\section{SANS}

Small-angle neutron scattering spectra of the purified normal and mutant NP particles were measured at 15,32 and $37^{\circ} \mathrm{C}$. Measurements were performed on a KWS-1 spectrometer (JCNS at MLZ, FRM-2 reactor, Garching, Germany) at three separate detector positions covering the $q$ range from 0.003 to $0.25 \AA^{-1}$. Gyration radius $R g$ and $P(R)$ function were calculated using ATSAS software (PRIMUSQT [16] and GNOM [17], respectively). Origin2015 software was used for visualization.

\section{Results and discussion}

In order to examine the role of the protein region containing the mutated residue in cold adaptation, we performed molecular dynamics simulations based on structures obtained by modification of a published structure (4BBL) [5]. In that published structure, it is clear that the amino acid residue that underwent substitution is located at the interface between double protein helix strands. We performed molecular dynamics of the mutant and normal proteins at two temperatures: $26^{\circ} \mathrm{C}$, the known lowest tolerable replication temperature for the mutant; and $39^{\circ} \mathrm{C}$, the

normal temperature for virus propagation. Figure 1 shows several aspects of NP structure: the left panel shows the representative structure obtained from the published RNP structural data [5]; the center panel details structures obtained as a result of molecular modeling, built into the same electron density distribution from the published RNP structure; and the right panel shows the position of amino acid residue 292E, which is substituted by G in NP from the coldadapted strain.

According to the published RNP structure (the
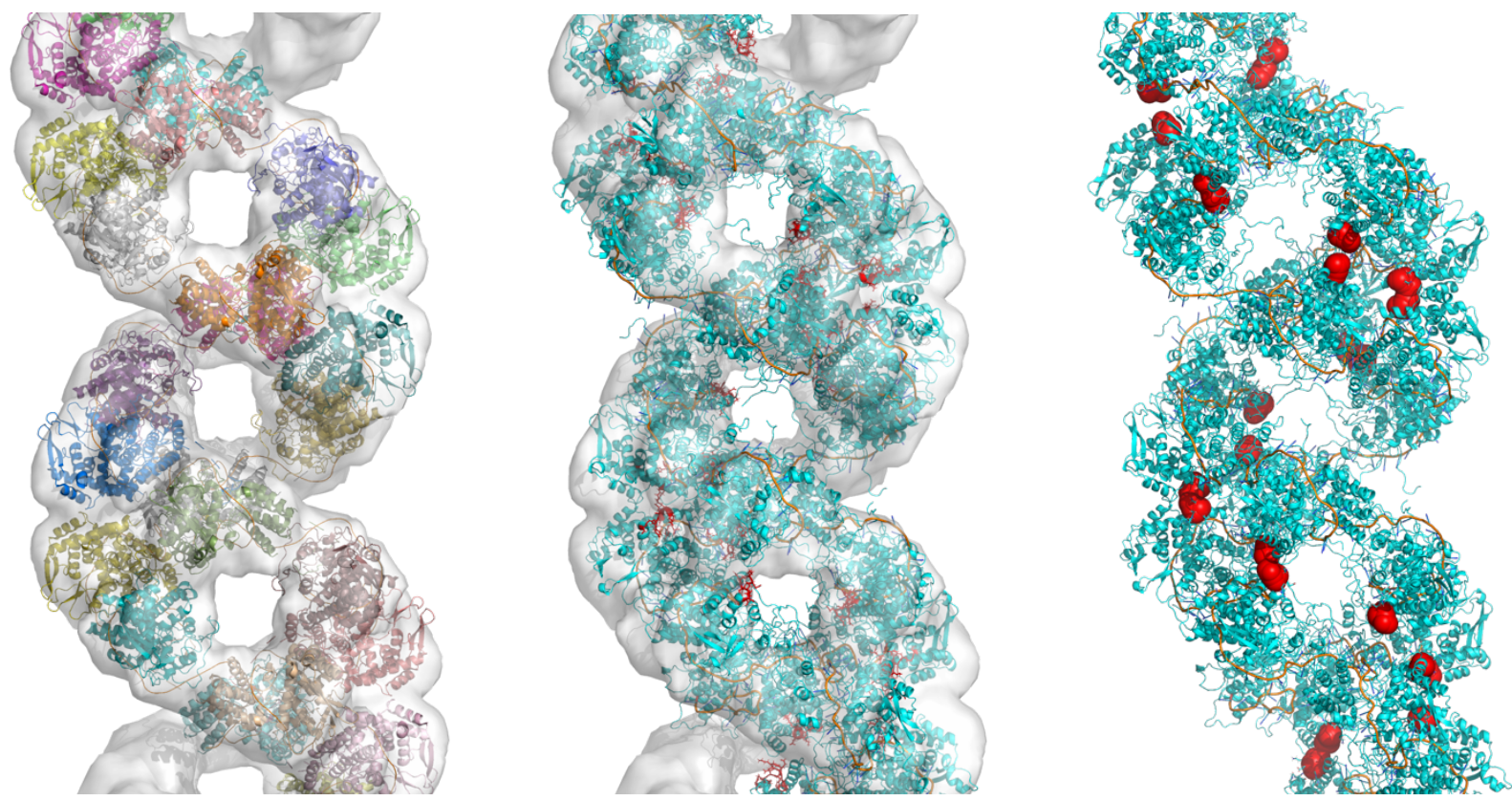

Figure 1. Several details of published and modeled NP structure (discussed in text). 
starting point for modeling dynamics), identical protein strand regions near position 292 are located opposite each other (Figure 1, right panel). Figure 2 shows an enlarged section of the ${ }^{287}$ SGYDFEREGYS ${ }^{297}$ protein fragment located near residue 292.

The distances between 292 residues of the adjacent monomers within the same chain, obtained by an analysis of four replicate MD trajectories, exhibited a large degree of scatter due to the filament structure's flexibility (Figure 3). While, on average, these distances were slightly shorter than those observed in the published RNP structure (63 $\AA$, denoted by horizontal lines in plots), these results show no evidence of steric interactions or any strong allosteric coupling in this set of monomer pairs. The distances do not show temperature dependency, nor do they undergo any significant changes in the mutant protein, as compared to wt (Figure 3).

Analysis of MD data on distances between the 292 residues of monomers located in opposing chains indicates a strong interaction between these regions in both wt and mutant protein. However, while the published structure implies an ordered filament with

(a)

WT @ 299K

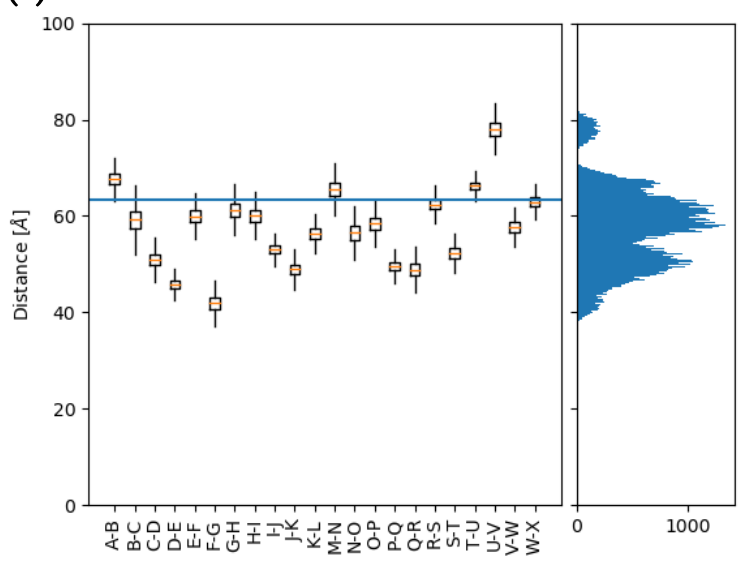

(c)

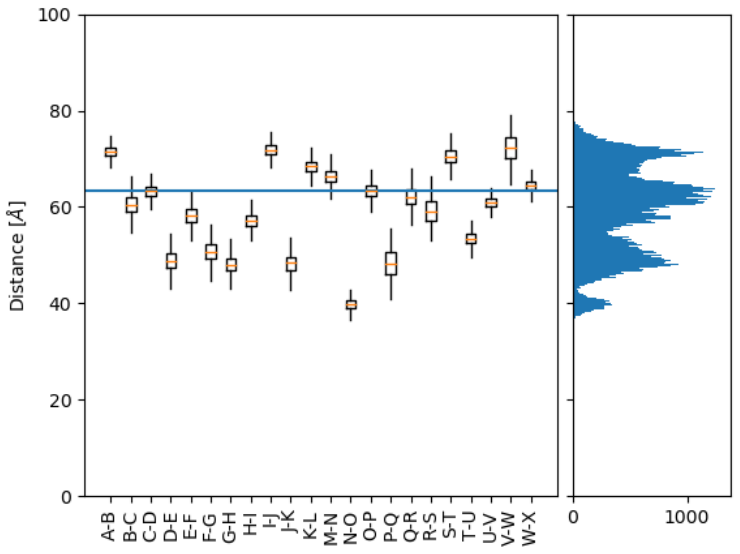

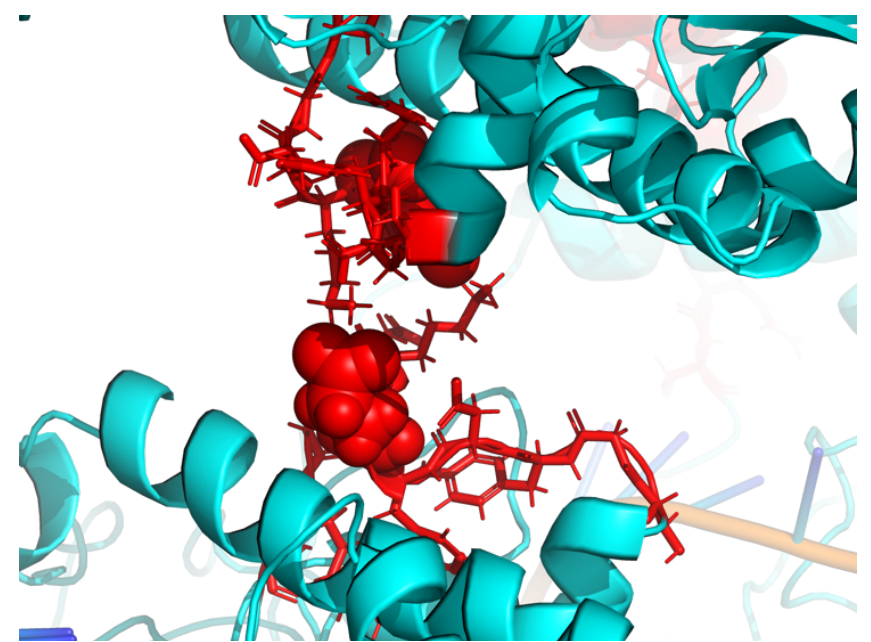

Figure 2. Enlarged rendering of the interface between ribonucleoprotein chains. The ${ }^{287}$ SGYDFEREGYS ${ }^{297}$ fragment is shown in red; the amino acid residue 292 (E) is shown in ball representation.

the distances in question of approximately $10 \AA$, only about half of these pairs maintained contact throughout our MD simulations (Figure 4). Raising the temperature from 26 to $39^{\circ} \mathrm{C}$ appears to stabilize some of the contacts, seen as decreased mean distance between the interacting residues. This may be

(b)

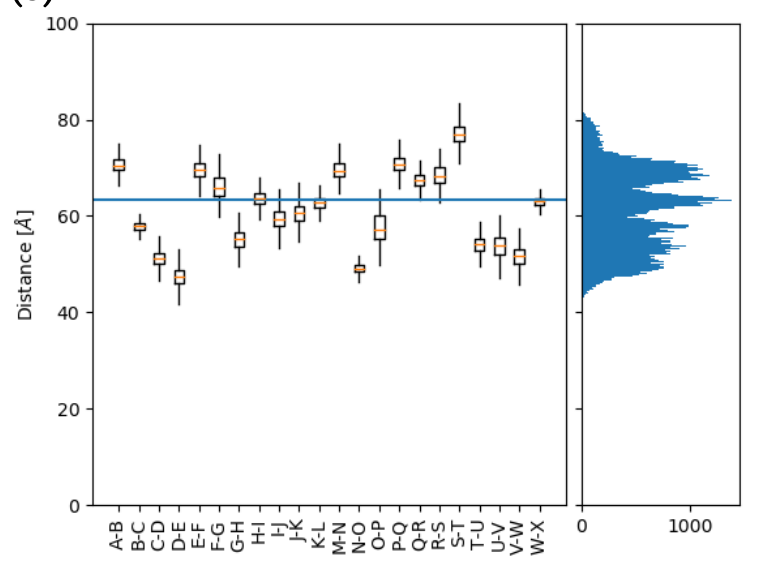

(d)

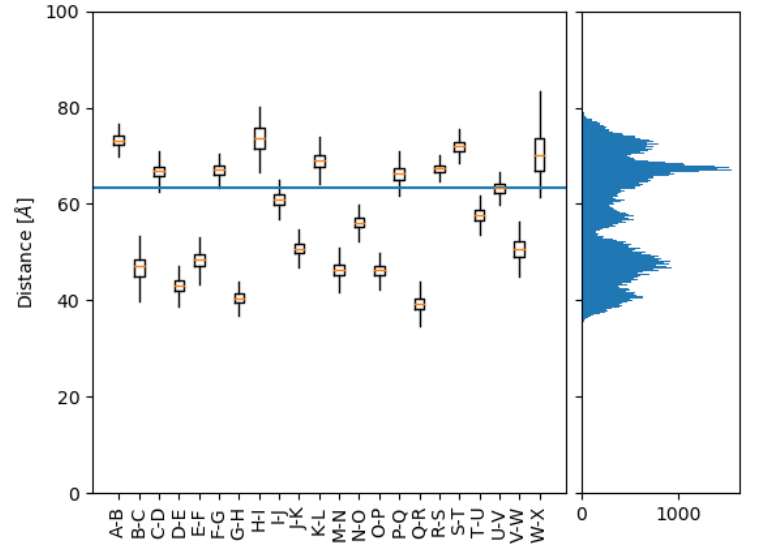

Figure 3. Distances between 292 residues within the same chain of wild-type (wt) and mutant (E292G) RNP at two temperatures: $26^{\circ} \mathrm{C}(299 \mathrm{~K})$ and $39^{\circ} \mathrm{C}(312 \mathrm{~K})$. The histograms on the right show the detailed distribution of distances (Counts vs Distance). 
(a)

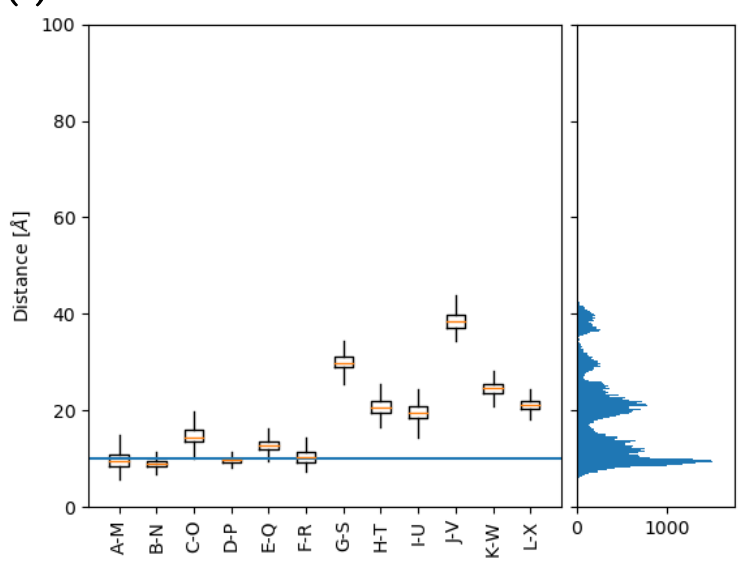

(c)

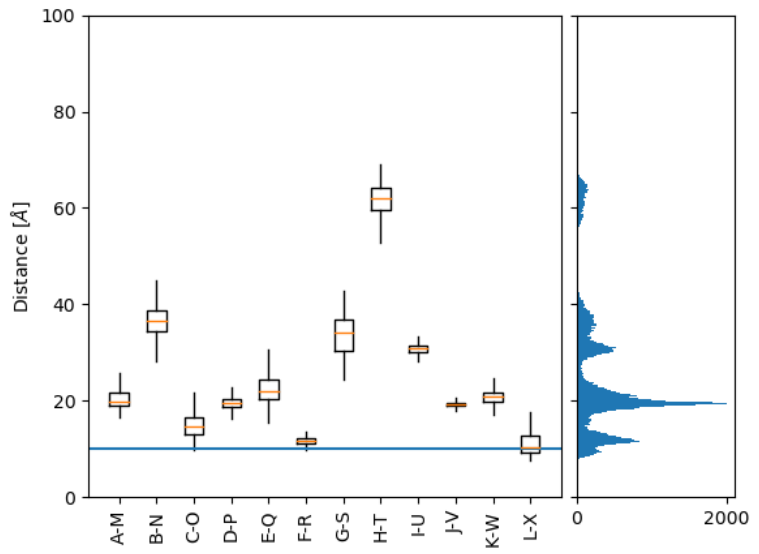

(b)

WT @ 312K

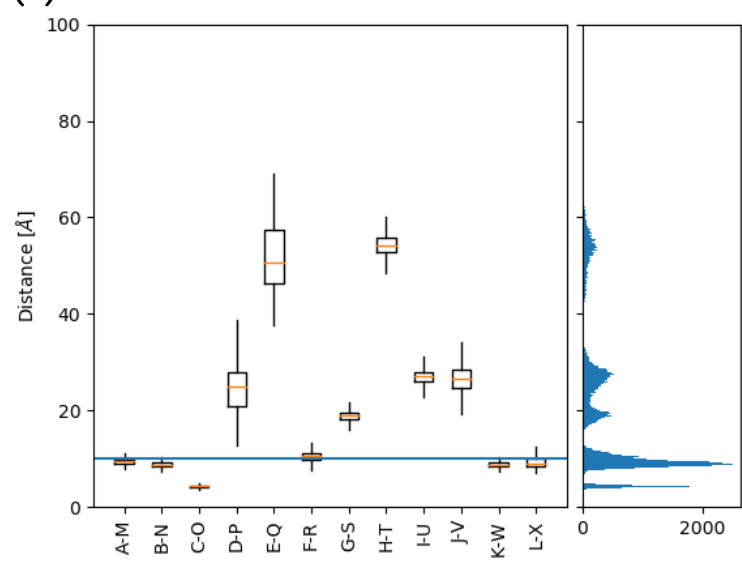

(d)

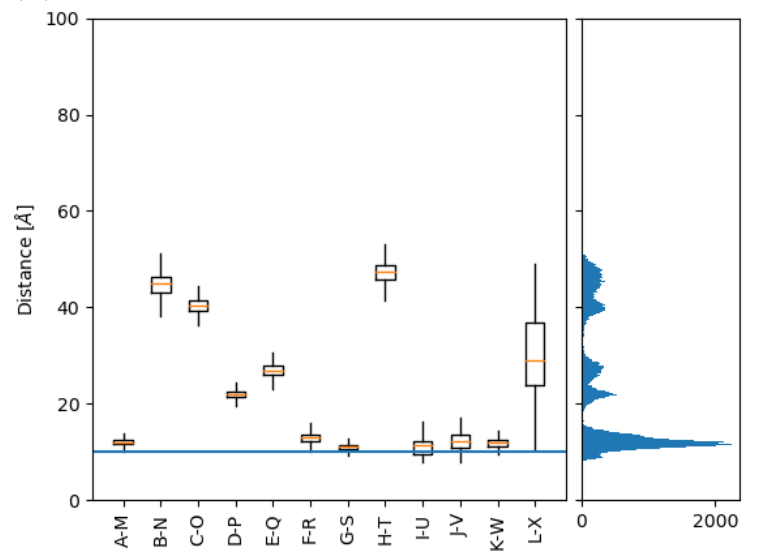

Figure 4. Distances between 292 residues in opposing chains of wild-type (wt) and mutant (E292G) RNP at two temperatures: $26^{\circ} \mathrm{C}(299 \mathrm{~K})$ and $39^{\circ} \mathrm{C}(312 \mathrm{~K})$. The histograms on the right show the detailed distribution of distances (Counts vs Distance).

mediated by alleviation of geometric constraints via increased large-scale filament flexibility at the higher temperature and emergence of pairs with very large distances (> $40 \AA$ ). The mutant protein filament was characterized by mean distances between 292 residues (on opposing chains) that were larger than in the wild type, as well as by the presence of pairs with distances over $40 \AA$ at both $26^{\circ} \mathrm{C}$ and $39^{\circ} \mathrm{C}$.

The detected increase in the distance variability between the double-stranded protein helix chains (at a lower temperature in the protein carrying the E292G substitution) allowed us to hypothesize that the cold adaptation phenotype depends on this structural feature of NP filaments. Functionally, this can be explained by known influenza A RNA-dependent RNA polymerase mechanisms. Specifically, regulation of interaction between protein strands in RNPs is critical for the operation of the enzyme [5]. In addition, the ability of the double protein helix to unwind and other significant changes in RNP helical structure have been shown [18]. We have previously published differences in RNP structure in solution at room temperature $\left(22^{\circ} \mathrm{C}\right)$ and operating temperature $\left(37^{\circ} \mathrm{C}\right)$, according to small-angle X-ray scattering data [15]. It is important to note that in different strains featuring different reproduction temperature characteristics, various molecular mechanisms may be behind the coldadapted phenotype. A detailed review of the currently known cold-adapted or temperature-sensitive strains, including information on amino acid substitutions that affect the ribonucleoprotein complex or temperature features, has been published [19]. In order to verify properties predicted by computer modeling, RNPs from mutant and the wild-type influenza strains were isolated, purified, and characterized. Isolations were carried out according to described methods [14] using centrifugation in glycerol density gradients. Electron micrographs of the obtained RNPs, formed by normal and mutant proteins, are shown in Figure 5.

Differential scanning fluorimetry can be used to support findings collected using molecular dynamics (temperature-dependent changes in RNP structure). Briefly, a change in the $350 / 330 \mathrm{~nm}$ tryptophan selffluorescence intensity ratio is observed over a temperature gradient from 15 to $95^{\circ} \mathrm{C}$. It signifies a change in the tryptophan micro-environment, as 


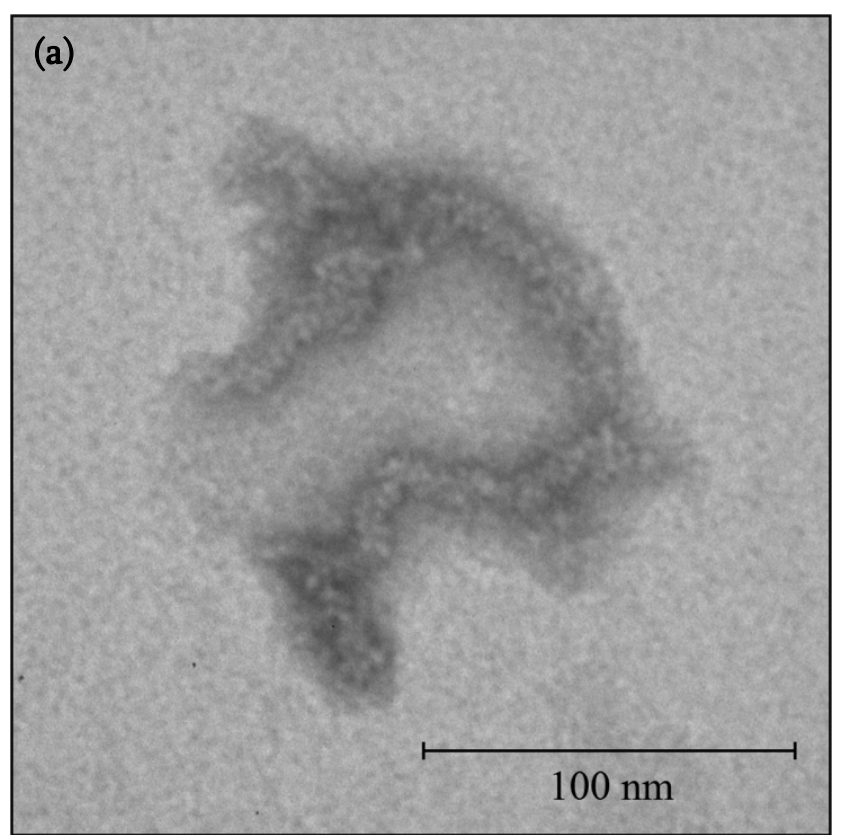

(b)

Figure 5. Electron microscopy of ribonucleoproteins isolated from normal (left) and mutant (right) protein-carrying strains.

dependent on temperature [20]. The experimental results are shown in Figure 6.

Analysis of the first derivative of the $350 / 330$ fluorescence intensity ratio shows the presence of a peak characteristic of NP (about $70^{\circ} \mathrm{C}$ ) [21]. Additional peaks, differing from each other, and corresponding to the local melting points of wild type and mutant protein-containing RNPs $\left(42.6^{\circ} \mathrm{C}\right.$ and $39.2^{\circ} \mathrm{C}$, respectively) were seen that can be attributed to partial double protein helix unwinding. The obtained results indicate an influence of the studied mutation on the temperature dependence of the RNP structure. However, the data do not permit pinpointing of the exact RNP structural element responsible for the changes observed. We assert that the changes in the distance between position 292 residues (between nucleoproteins of opposing RNP chains), observed during simulation, are related to the temperature dependence of the interaction at the corresponding interfaces (shown in Figure 2). To test the hypothesis that interactions at the interface play a role, not allosteric effects acting on the chains from elsewhere, we applied an interface peptide model. A commercial NP protein from influenza H3N2, which does not carry the E292G substitution, was immobilized onto a chip. The interaction between chip and either of two
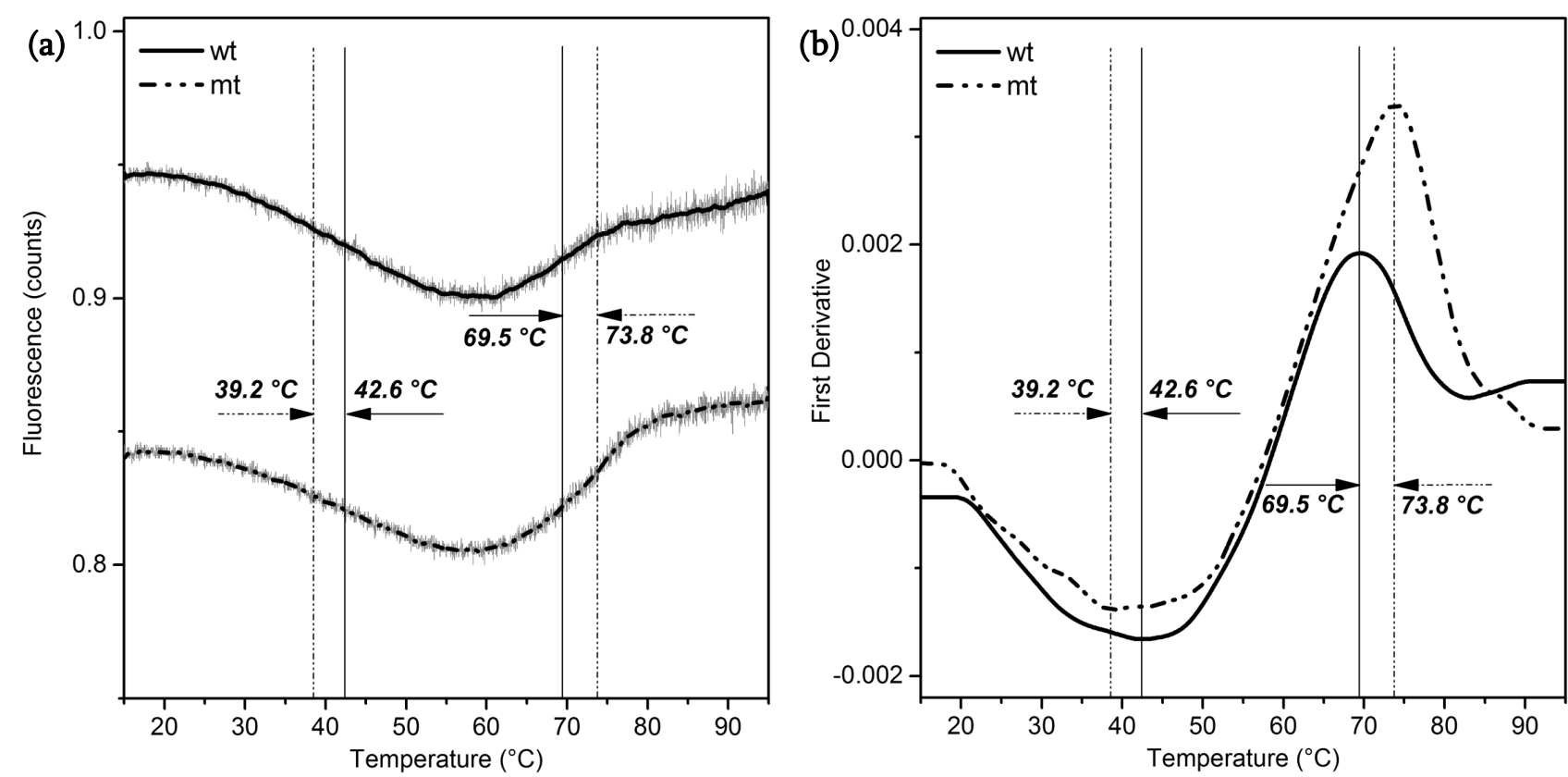

Figure 6. Differential scanning fluorimetry (DSF): (a) intrinsic fluorescence (350/330 nm ratio) of tryptophan as a function of temperature in samples containing wild-type (wt) or E292G mutant (mt) RNP; (b) first derivative of (a). 

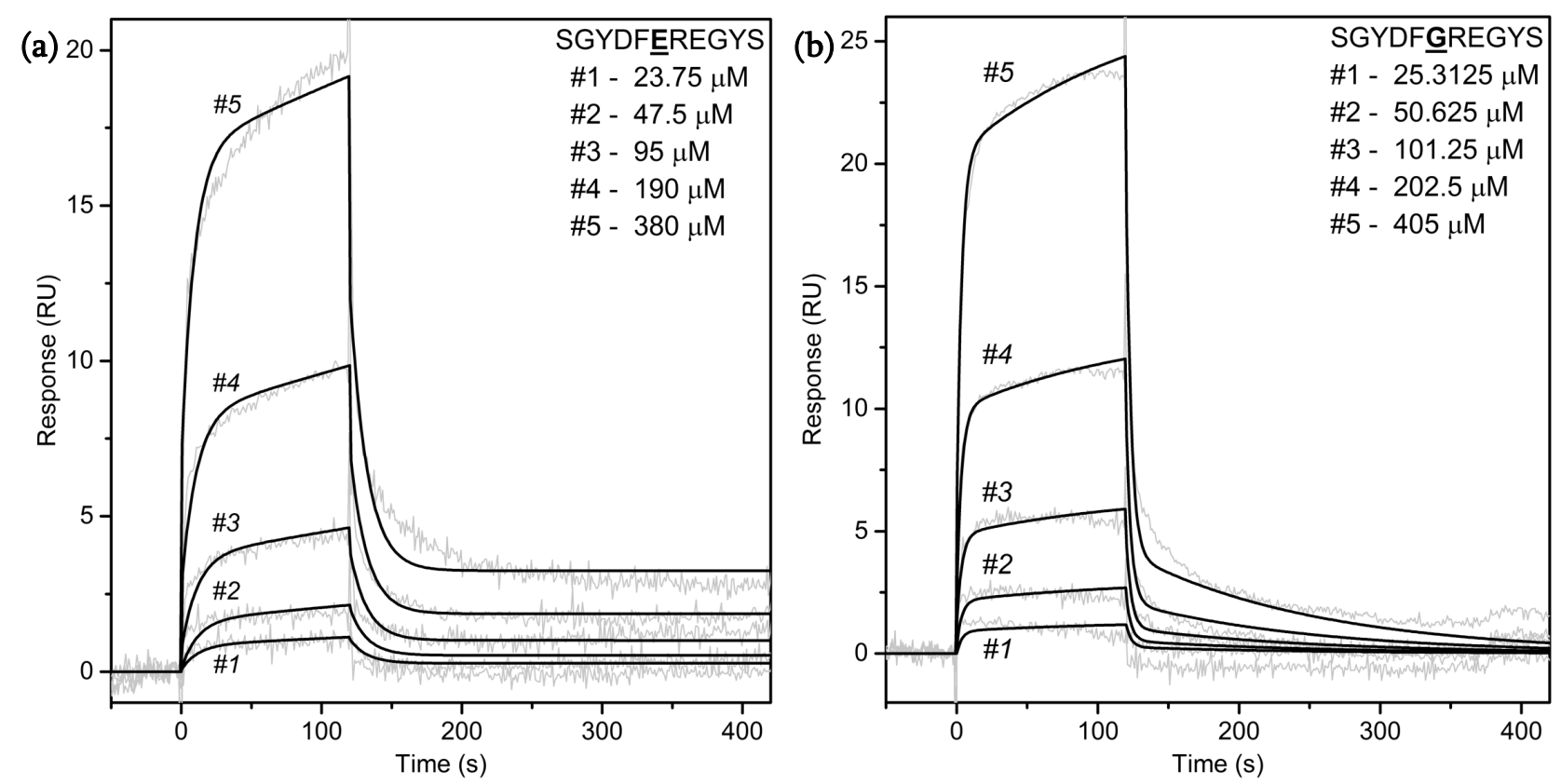

Figure 7. Sensograms of the interaction of peptides, whose primary structures correspond to wt and mutant (E292G) NP protein interfaces, with monomeric NP immobilized on chip; surface plasmon resonance data.

Table 1. Constants determined by the two-step reaction model.

\begin{tabular}{|l|c|c|c|c|c|}
\hline & $\mathrm{k}_{\mathrm{a} 1}(1 / \mathrm{Ms})$ & $\mathrm{kd}_{1}(1 / \mathrm{s})$ & $\mathrm{k}_{\mathrm{a} 2}(1 / \mathrm{s})$ & $\mathrm{kd}_{2}(1 / \mathrm{s})$ & $\mathrm{KD}(\mathrm{M})$ \\
\hline SGYDFEREGYS & 71.48 & 0.0799 & 0.0027 & $1.54 \mathrm{E}-06$ & $6.32 \mathrm{E}-07$ \\
\hline SGYDFGREGYS & 17.09 & 0.2899 & 0.0032 & $\mathbf{0 . 0 0 7 6}$ & 0.0115 \\
\hline
\end{tabular}

peptides (SGYDFEREGYS or SGYDFGREGYS), which are peptide analogues of the RNP protein strand interaction interface, was studied by surface plasmon resonance (Figure 7).

The macromolecular interaction data are best modeled mathematically (Table 1 ) using a two-step reaction model [22]:

$$
\mathrm{A}+\mathrm{B} \underset{\mathrm{k}_{\mathrm{d} 1}}{\stackrel{\mathrm{k}_{\mathrm{a} 1}}{\rightleftarrows}} \underset{\mathrm{k}_{\mathrm{d} 2}}{\stackrel{\mathrm{k}_{\mathrm{a} 2}}{\rightleftarrows}} \mathrm{AB}^{*},
$$

where $\mathrm{k}_{\mathrm{a}}$ - association rate constant, $\mathrm{k}_{\mathrm{d}}$ - dissociation rate constant, $\mathrm{K}_{\mathrm{D}}$ - equilibrium dissociation constant.

The only significant difference between the study peptides, in terms of this model, was the reverse reaction constant $k_{d} 2\left(A B^{*}\right.$ to $\left.A B\right)$. Specifically, in the case of the SGYDFEREGYS (wild-type) peptide, this reverse transformation is slow. However, with the SGYDFGREGYS (E292G mutant) peptide, the forward and backward reactions rates are comparable. Thus, it turns out that the unsubstituted peptide is more prone to interaction with the full-sized immobilized protein. At a fixed temperature $\left(25^{\circ} \mathrm{C}\right)$, the interactions between the immobilized protein and peptides from solution were different, which may be due to the role of $\mathrm{E}$ at position 292 in interface interactions. We also performed an analysis of study peptides in terms of temperature-dependent transition from monomeric to oligomeric (aggregated) form. Figure 8 shows the temperature dependence of $540 \mathrm{~nm}$ absorption for the SGYDFEREGYS and SGYDFGREGYS peptides.

In other words, the peptide corresponding to the wild-type sequence portion showed a tendency to selfassociation at temperatures above 30 degrees, while the one carrying the substitution did not aggregate. The presence of temperature-dependent oligomerization ability in the wt model peptide likely indicates a role

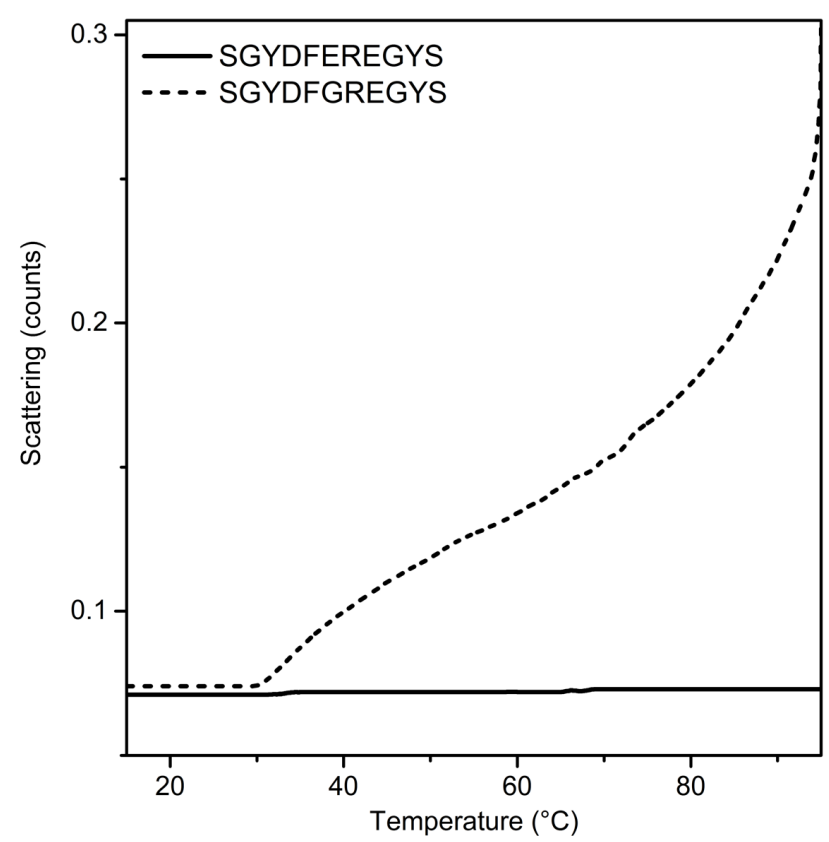

Figure 8. Temperature dependence of $540 \mathrm{~nm}$ absorption of peptide solutions at a concentration of $0.7 \mathrm{mg} / \mathrm{ml}$. 
of the glutamic acid residue at position 292 in selfassociation. In the peptide model, the presence of a substitution at the interface between the chains significantly affects: (i) the ability to interact with a protein containing an identical primary structure fragment; and (ii) the propensity for temperaturedependent self-association. These facts indirectly indicate a role of the glutamic acid residue at position 292 in the interface interaction during formation of the nucleoprotein complex.

To investigate the effect of E292G in temperaturedependent ribonucleoprotein complex structural changes, we performed small angle neutron scattering (SANS) measurements of the structures of RNPcontaining wild-type and mutant protein (Figure 9).

SANS shows that, at three temperatures $\left(15^{\circ} \mathrm{C}, 32^{\circ} \mathrm{C}\right.$, $37^{\circ} \mathrm{C}$ ), RNP structures form from both wt and mutant proteins leading to different spectra. At $15^{\circ} \mathrm{C}$, the SANS data can be interpreted using a model featuring strongly elongated particles with a cross-sectional gyration radius of about $40 \AA$ for the wt and of $50 \AA$ for the RNP formed from substitution-carrying protein (Figure 9a, inset). These results match the published RNP double helix structure from the H1N1 influenza virus [5]. Temperature increases (from $15^{\circ} \mathrm{C}$, to $32^{\circ} \mathrm{C}$, and to $37^{\circ} \mathrm{C}$ ) led to significant changes in SANS spectra, and while the behavior of the curves at low q was still close to the 1/q, noticeable deviations from the Guinier approximation for rod-like particles at small angles were observed, accompanied by an increase in the scattering of the samples in the $\mathrm{q}$ range between 0.01 and $0.03 \AA^{-1}$. (Figure 9a, inset).

The distance distribution functions calculated from the experimental SANS spectra of RNP (Figure $9 \mathrm{~b}$ and c) show two distinct maxima at $4-5 \mathrm{~nm}$ and $15 \mathrm{~nm}$. We have performed an analysis of the mean distance distribution between the centers of mass of all the monomer pairs in the model filament, averaged over the MD trajectory. Figure 10 shows the results of that calculation, with the pairs of monomers which would contribute to the experimentally observed peaks highlighted in the bottom-right half of each panel. Distances of approx. $5 \mathrm{~nm}$ were observed between the

Figure 9. Small angle neutron scattering of wild-type (wt) and E292G mutant (mt) RNP solutions at $15^{\circ} \mathrm{C}, 32^{\circ} \mathrm{C}$, and $37^{\circ} \mathrm{C}$. (a) Representation in double logarithmic scale $I$ vs $q$, where $I$ - scattering intensity, $q$ - magnitude of the momentum transfer; inset - Guinier coordinates ( $I q$ vs $\left.q^{2}\right)$. Distance distribution function $P(R)$, calculated for SANS spectra of wild-type RNP (b) and E292G mutant (c); dash lines mark $R=50$ and $150 \AA$.
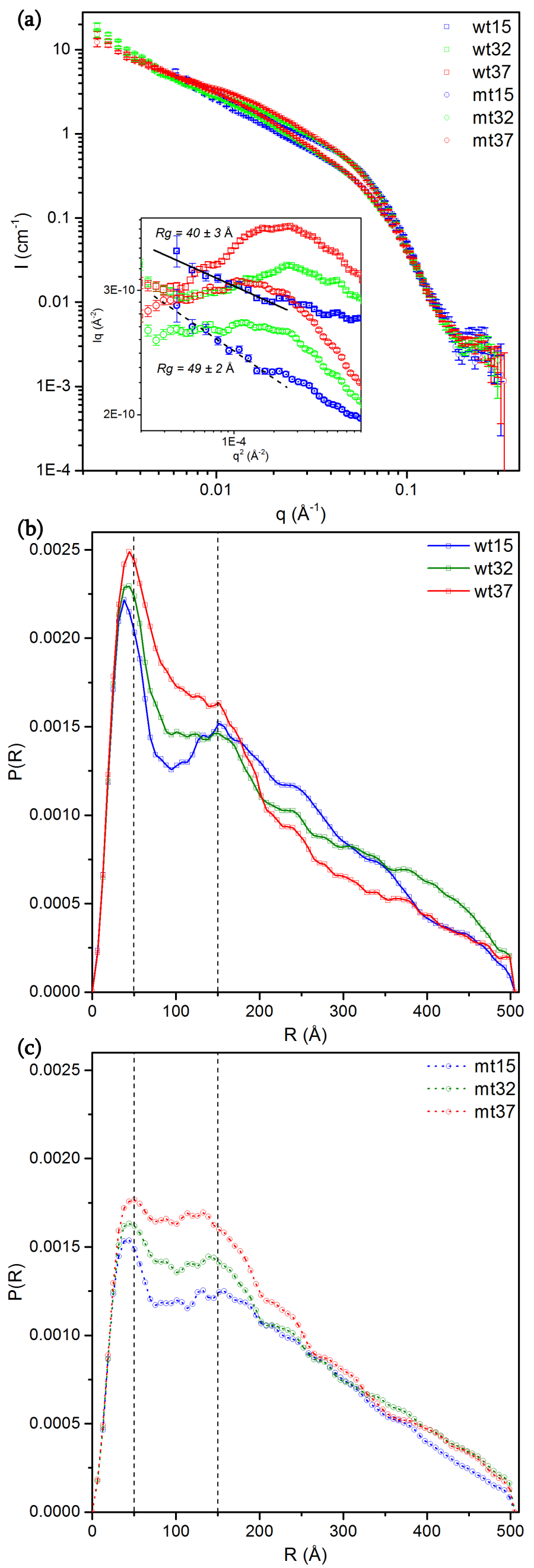
adjacent monomers along the same protein chain and also between the interacting monomers of the two filaments in the double helix. The distance of $15 \mathrm{~nm}$ corresponds to the pairs of monomers separated by one turn of the helix (Figure 11). It should be noted that, unlike the reference double-stranded helical nucleoprotein complex structure [5], in the $\mathrm{MD}$ simulated structure: both pair-distance measurement types (cis - on monomers of the same nucleoprotein complex strand; trans - on different strands, separated by 1 turn in the nucleoprotein complex helix) fall into

(a)

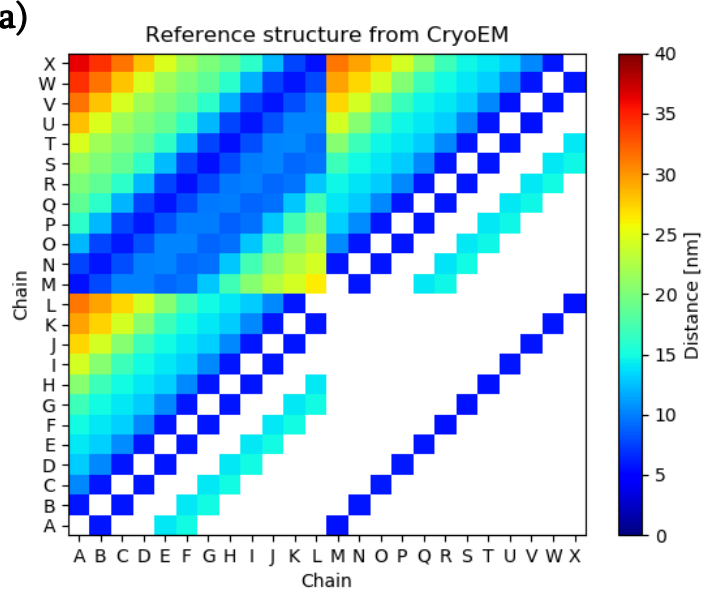

(b)

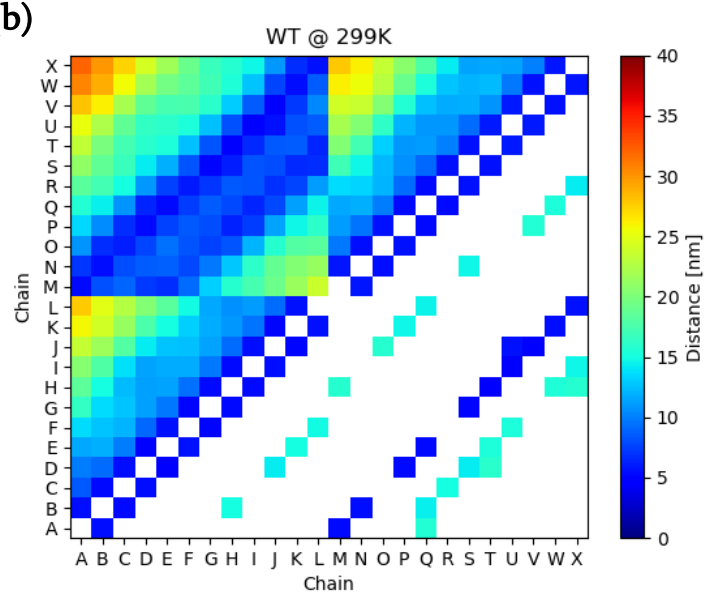

(d)

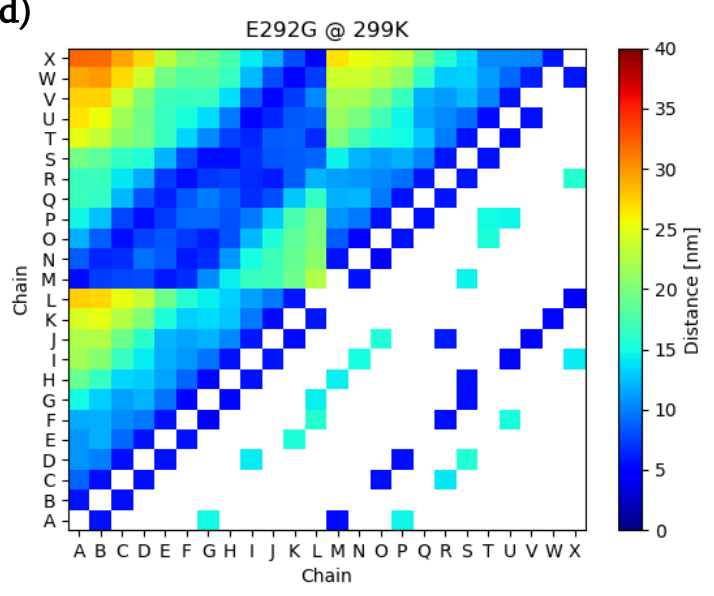

the range $14-16 \mathrm{~nm}$, thus giving rise to a peak in the distance distribution function [5].

The temperature-dependent differences in SANS of wild type and mutant RNP highlight structural differences between them. The mutant protein appears to form fewer contacts between potentially interacting regions of adjacent NP filaments. Loss of RNP's rod-like shape at elevated temperatures, accompanied by a shift of the first $\mathrm{P}(\mathrm{R})$ peak toward larger distances (from 4 $\mathrm{nm}$ to approx. $5 \mathrm{~nm}$ ), may be attributable to decreased interaction between regions in two adjacent NP filaments, leading to large distortions in RNP structure.

Figure 10. Mean distance distribution between the centers of mass of all monomer pairs, calculated for the reference structure [5] (a), for wild-type (wt), and for E292G mutant (mt) NP at $26^{\circ} \mathrm{C}(299 \mathrm{~K})$ and $39^{\circ} \mathrm{C}(312 \mathrm{~K})(\mathrm{b}-\mathrm{e})$. "Chains" from A to L correspond to NP monomers in the first helix chain of RNP; those from $\mathrm{M}$ to $\mathrm{X}$ correspond to the second

(c)

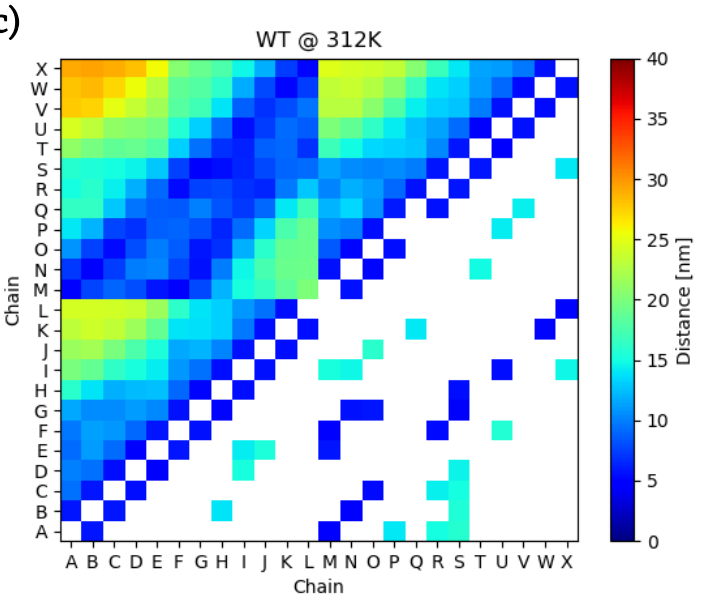

(e)

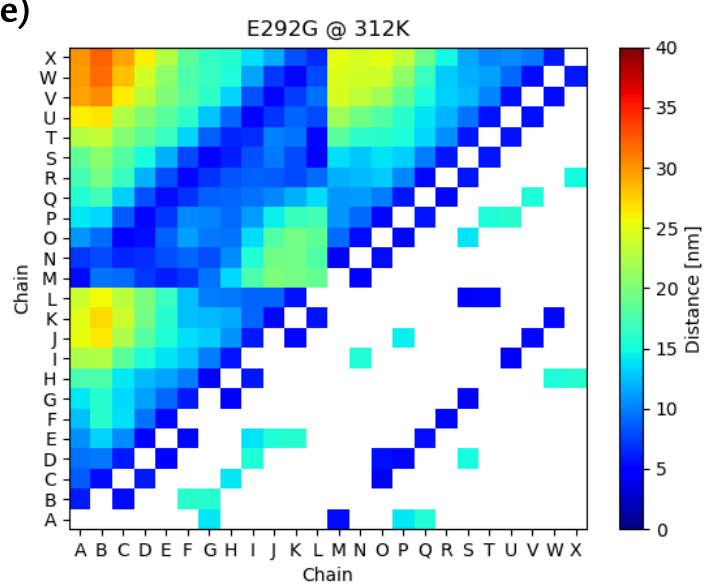




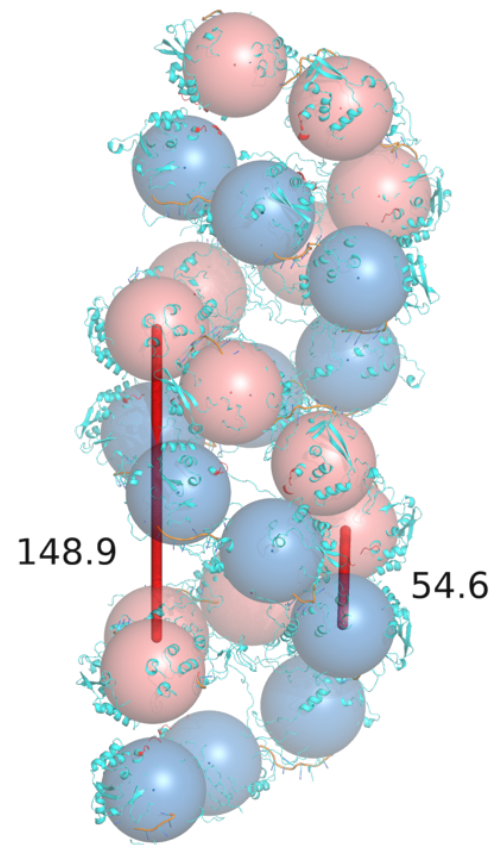

Figure 11. Model of RNP double-helix filament. Blue and red balls represent NP monomers. The characteristic distances between the monomers' centers of mass (148.9 and 54.6 ̊) are indicated by red bars.

\section{Conclusions}

Molecular dynamics simulation data, together with DSF and SANS results, suggest high structural lability of the NP filament; this likely permits partial unwinding of portions of the protein double helix near functional temperatures, a necessary step for RNA polymerase function. Using a peptide model, the region responsible for the strand interaction has been confirmed to contain E292 the residue. Apparently, the presence of amino acid substitution E292G, which is associated with cold adaptation, weakens interactions between NP strands in RNPs, thereby making possible their partial dissociation at lower temperature.

\section{Acknowledgments}

This work was supported by Russian Science Foundation grant № 19-74-20146. The SANS experiments were performed on KWS-1 (proposal number 7879) and KWS-2 (proposal number 9634). The Prometheus PR.48 equipment used for nanoDSF experiments was provided by Nanotemper Technologies RUS LLC.

\section{Bibliography}

[1] "WHO | Influenza (Seasonal)," 2018. [Online]. Available: https://www.who.int/news-room/factsheets/detail/influenza-(seasonal).

[2] A. S. Gambaryan et al., "Comparative safety, immunogenicity, and efficacy of several anti$\mathrm{H} 5 \mathrm{~N} 1$ influenza experimental vaccines in a mouse and chicken models (Testing of killed and live H5 vaccine)," Influenza Other Respi. Viruses, vol. 6, no. 3, pp. 188-195, May 2012.

[3] L. M. Tsybalova et al., "[Characterization of Coldadapted Influenza Strain a/Hong Kong/1/68/162/35 as a Potential Donor of Attenuation and High Reproduction]," Vopr. Virusol., vol. 57, no. 6, pp. 13-17, 2012.

[4] A. Vasin, O. Temkina, S. Klotchenko, M. Plotnikova, V. Egorov, and O. Kiselev, "Molecular mechanisms enhancing the coding potential of RNA genome of influenza A viruses," FEBS Journal (38th Congress of the Federation of European Biochemical Societies), vol. 280, no. s1, St. Petersburg, p. 43, 2013.

[5] R. Arranz et al., "The structure of native influenza virion ribonucleoproteins," Science (80-. )., vol. 338, no. 6114, pp. 1634-1637, Dec. 2012.

[6] A. A. Pulkina et al., "Impact of mutations in nucleoprotein on replication of influenza virus A/Hong Kong/1/68/162/35 reassortants at different temperatures," Mol. Biol., vol. 51, no. 2, pp. 333-

\section{8, Mar. 2017.}

[7] Q. Ye, R. M. Krug, and Y. J. Tao, "The mechanism by which influenza A virus nucleoprotein forms oligomers and binds RNA," Nature, vol. 444, no. 7122, pp. 1078-1082, Dec. 2006.

[8] W. L. DeLano, "The $\{$ PyMOL $\}$ Molecular Graphics System, Version 1.8," Schrödinger, LLC, 2015.

[9] M. J. Abraham et al., "GROMACS: High performance molecular simulations through multi-level parallelism from laptops to supercomputers," SoftwareX, vol. 1-2, pp. 19-25, Sep. 2015.

[10] H. J. C. Berendsen, D. van der Spoel, and R. van Drunen, "GROMACS: A message-passing parallel molecular dynamics implementation," Comput. Phys. Commun., vol. 91, no. 1-3, pp. 43-56, Sep. 1995.

[11] K. Lindorff-Larsen et al., "Improved side-chain torsion potentials for the Amber ff99SB protein force field," Proteins Struct. Funct. Bioinforma., vol. 78, no. 8, pp. 1950-8, Jun. 2010.

[12] A. Pérez et al., "Refinement of the AMBER force field for nucleic acids: Improving the description of $\alpha / \gamma$ conformers," Biophys. J., vol. 92, no. 11, pp. 3817-3829, 2007.

[13] W. L. Jorgensen, J. Chandrasekhar, J. D. Madura, R. W. Impey, and M. L. Klein, "Comparison of simple potential functions for simulating liquid 
water," J. Chem. Phys., vol. 79, no. 2, pp. 926-935, Jul. 1983.

[14] W. W. H. Wu, L. L. Weaver, and N. Panté, "Purification and Visualization of Influenza A Viral Ribonucleoprotein Complexes," J. Vis. Exp., vol. 10, no. 10, p. E560, Feb. 2009.

[15] V. V. Egorov et al., "On the structural features of influenza A nucleoprotein particles from smallangle X-ray scattering data," J. Surf. Investig. $X$ ray, Synchrotron Neutron Tech., vol. 10, no. 2, pp. 322-325, Mar. 2016.

[16] P. V. Konarev, V. V. Volkov, A. V. Sokolova, M. H. J. Koch, and D. I. Svergun, "PRIMUS: A Windows PC-based system for small-angle scattering data analysis," J. Appl. Crystallogr., vol. 36, no. 5, pp. 1277-1282, Oct. 2003.

[17] D. I. Svergun, "Determination of the regularization parameter in indirect-transform methods using perceptual criteria," J. Appl. Crystallogr., vol. 25, no. pt 4, pp. 495-503, Aug. 1992.
[18] J. R. Gallagher, U. Torian, D. M. McCraw, and A. K. Harris, "Structural studies of influenza virus RNPs by electron microscopy indicate molecular contortions within NP supra-structures," J. Struct. Biol., vol. 197, no. 3, pp. 294-307, Mar. 2017.

[19] L. Martínez-Sobrido, O. Peersen, and A. Nogales, "Temperature sensitive mutations in influenza a viral ribonucleoprotein complex responsible for the attenuation of the live attenuated influenza vaccine," Viruses, vol. 10, no. 10. MDPI AG, p. E560, 15-Oct-2018.

[20] "Prometheus NT.48 - TechnoInfo," 2018. [Online]. Available: https://technoinfo.co.uk/catalog/prometheus-nt48/. [Accessed: 04-Nov-2019].

[21] S. Chenavas et al., "Monomeric Nucleoprotein of Influenza A Virus," PLoS Pathog., vol. 9, no. 3, p. e1003275, Mar. 2013.

[22] Y. A. Zabrodskaya et al., "The amyloidogenicity of the influenza virus PB1-derived peptide sheds light on its antiviral activity," Biophys. Chem., vol. 234, pp. 16-23, 2018. 